\title{
Microarray analysis reveals differential expression of benign and malignant pheochromocytoma
}

\author{
Jens Waldmann ${ }^{1}$, Volker Fendrich ${ }^{1}$, Julia Holler ${ }^{6}$, Malte Buchholz ${ }^{2}$, \\ Ernst Heinmöller, ${ }^{3}$, Peter Langer ${ }^{1}$, Annette Ramaswamy ${ }^{4}$, Birgit Samans ${ }^{5}$, \\ Martin K Walz ${ }^{7}$, Matthias Rothmund ${ }^{1}$, Detlef $K$ Bartsch $^{1}$ and Emily $P$ Slater ${ }^{1}$ \\ ${ }^{1}$ Department of Surgery and ${ }^{2}$ Division of Gastroenterology, Philipps-Universität Marburg, Baldingerstraße, D-35043 Marburg, \\ Germany \\ ${ }^{3}$ Department of Pathology, Klinkum Kassel, 34125 Kassel, Germany \\ ${ }^{4}$ Department of Pathology, Philipps-Universität Marburg, Baldingerstraße, D-35043 Marburg, Germany \\ ${ }^{5}$ Institute of Molecular Biology and Tumor Research, Philipps-University Marburg, 35037 Marburg, Germany \\ ${ }^{6}$ Department of Surgery, University Giessen, 35392 Giessen, Germany \\ ${ }^{7}$ Department of Surgery, Center for Minimally Invasive Surgery, Kliniken Essen-Mitte, 45136 Essen, Germany \\ (Correspondence should be addressed to J Waldmann; Email: jwaldman@ @med.uni-marburg.de)
}

\begin{abstract}
The diagnosis of a malignant pheochromocytoma (PC) can only be established by the presence of distant metastases, but a subset of apparently benign PCs develop metastases. We have employed a microarray analysis to identify a typical gene expression profile which distinguishes malignant from benign PC. Total RNA was isolated from fresh-frozen tissue of five benign and five malignant PCs. The reference consisted of laser microdissected tissue from normal adrenal medulla. After generating Cy3- and Cy5-fluorescently labeled cDNAs, F-chips containing 11540 spots were hybridized. Data were analyzed with the IMAGENE 3.0 software. Gene expression levels were validated by real-time (RT)-PCR and immunohistochemistry (IHC). The analysis revealed a more than twofold difference in expression between benign and malignant PCs in 132 genes: 19 were up-regulated and 113 were down-regulated. Expression differences of six genes (calsequestrin, NNAT, neurogranin, secreted protein acidic and rich in cysteine (SPARC), EGR2, and MAOB) were confirmed by RT-PCR in 25 PCs. IHC for calsequestrin revealed an overexpression in malignant PCs $(7 / 10$ vs $1 / 10, P=0.03)$. Comparative analysis by microarray of all ten PCs (benign/malignant) versus normal adrenal medulla revealed a more than twofold expression difference in 455/539 and 491/671 genes respectively. Several of these genes are known to participate on adrenal tumorigenesis, potential tumor suppressor genes, and oncogenes. Comprehensive gene expression analysis of malignant and benign PCs revealed different gene profiles, which could be used to discriminate between malignant and benign PCs. Based on these findings, the strategy for further follow-up and treatment could be modified accordingly.
\end{abstract}

Endocrine-Related Cancer (2010) 17 743-756

\section{Introduction}

Pheochromocytomas (PCs) are rare neoplasms of the adrenal medulla, which are derived from chromaffin cells of the neural crest. Extra-adrenal PC or paraganglioma (PG) arise from closely related chromaffin cells in sympathetic or parasympathetic paraganglia. The annual incidence is $\sim 1 / 100000$ patient years, and PC causes secondary hypertension in about $1 \%$ (Beard et al. 1983, Sinclair et al. 1987, Omura et al. 2004), although a prevalence of $0.05 \%$ is reported in autopsy studies (Platts et al. 1995, McNeil et al. 2000, Khorram-Manesh et al. 2004). Sporadic PCs are three times more frequent than the familial form of PC, which is a result of germline mutations affecting cancer susceptibility genes. Germline mutations affect the oncogene RET and the oncosuppressors $V H L, S D H B$, $S D H D$, and NF1. These familial PCs represent $\sim 25 \%$ of cases, and are part of the MEN2 syndrome, the PG 
syndromes 1, 3, and 4, the von Hippel-Lindau disease, and neurofibromatosis (Neumann et al. 2002). In the case of an inherited tumor syndrome, one must consider the higher rate of malignancy (most commonly associated with SDHB mutation), recurrence, and bilateral disease before patients are scheduled for surgery, which emphasizes the need for genetic testing in every patient with PC.

The surgical treatment of unilateral sporadic PC is well established, and laparoscopic adrenalectomy has become safe in the background of an adequate $\alpha$-blockage. The management of patients with bilateral disease is still unsettled. An indefinite follow-up is suggested by the NIH consensus conference for familial cases, and at least a 10-year follow-up is warranted for sporadic cases as recurrent and malignant diseases occur in about 17\% (Pacak et al. 2001, Amar et al. 2005). The major problem regarding the clinical management is the identification of patients with malignant disease before they develop distant metastases (DM) or recurrence as malignant PC is incurable.

The prevalence of malignancy in PC ranges from 2.4 to $26 \%$, depending on the definition of malignancy (Melicow 1977, Proye et al. 1992). Although the study of Proye et al. apparently overestimated the rate of malignancy by defining microscopic venous thrombi and regional invasion as true signs of malignancy. Only 14/91 (15\%) patients revealed DM. The highest rate of malignancy is observed in patients with SDHB mutations, and reaches 50\% (Gimenez-Roqueplo et al. 2003). However, the histological distinction of benign from malignant PC is not reliable. Malignancy is only proven by the presence of distant or lymph node metastases. Efforts have been made to establish molecular markers for malignancy such as overexpression of HSP90, human telomerase reverse transcriptase, HIF- $2 \alpha$, tenascin, N-cadherin, COX-2, and others. Comparative genomic hybridization experiments in malignant and benign $\mathrm{PC}$ revealed losses of $1 \mathrm{p}, 3 \mathrm{q}$, and $6 \mathrm{q}$, and gains of $9 \mathrm{q}$ and $17 \mathrm{q}$. Progression to malignant $\mathrm{PC}$ was associated with deletions on 6q and 17p (Dannenberg et al. 2000).

A recent microarray analysis of rat PC compared with normal adrenal medulla revealed numerous differentially expressed genes and $\sim 60 \%$ of these overlap with a human PC database (Elkahloun et al. 2006). Following the study from Giordano who initially assessed gene expression profiles of malignant and benign PC, five other groups have described similar studies (Eisenhofer et al. 2004a,b, Dahia et al. 2005, Brouwers et al. 2006, Thouennon et al. 2007). These clearly identified a set of genes that could be used as multimarkers for malignancy. Over $80 \%$ of differentially expressed genes were down-regulated, which led to the hypothesis that the malignant potential is related to a less differentiated expression pattern. Interestingly, Tischler found that PCs in Nf1 knockout mice express genes which are typical for neural progenitor cells (Powers et al. 2007).

Despite these data, the development of malignancy, tumorigenesis, and its underlying molecular basis are poorly understood.

In this study, we compared five benign and five malignant PCs to normal human adrenal medulla by cDNA microarrays to identify differentially expressed genes that discriminate malignant PC from benign PC and to improve our knowledge of underlying molecular mechanisms.

\section{Patients and methods}

\section{Patients and tumors}

All ten patients with PC included in this study underwent adrenalectomy in the Department of Surgery, University of Marburg between 1998 and 2006. The tumor tissue was frozen in liquid nitrogen 10-20 min after surgical removal, and stored in our collection of fresh-frozen adrenal tissue.

This study was performed according to the local ethics committee guidelines, and all patients gave their informed consent in written form.

For the purpose of this study, we chose five benign PCs and five malignant PCs. We only used sporadic benign PCs where a germline mutation had been excluded by mutation analysis (see below). Malignant PC tumor tissue was only available in five cases. The diagnosis of a malignant $\mathrm{PC}$ was based on the presence of DM. Frozen tumor samples were formalin-fixed, embedded in paraffin, and sections were evaluated with hematoxylin and eosin (HE) staining to determine the diagnosis and the neoplastic cellularity. To exclude contamination with normal adrenal medulla or cortical tissue, only tumor samples with a neoplasticity of at least $90 \%$ were subjected to further analysis.

In total, we analyzed 36 different primary PC samples: 10 in the microarray analysis, 25 by realtime (RT)-PCR, and 20 by immunohistochemistry (IHC). Adrenal tumors were classified as PCs, and extradrenal tumors were classified as PG.

\section{Normal control}

Normal adrenal medulla that served as the normal control consisted of 60 fresh-frozen sections from ten different patients who underwent adrenal surgery for 
reasons other than PC (five Conn's syndrome and five Cushing's syndrome). They were chosen randomly from our tissue bank that contains over 200 frozen samples of adrenal tumors and normal adrenal tissue. From ten slides of each normal adrenal tissue, we collected the adrenal medulla cells by laser microdissection (LMD) using a P.A.L.M. MicroBeam (Bernried, Germany).

\section{Laser microdissection}

Owing to the irregular distribution of adrenal medulla and cortex in an adrenal specimen, we performed a LMD to prevent cortical contamination in the normal medulla control. We stained six frozen sections $(10-\mu \mathrm{m}$ thickness) of each normal adrenal tissue in methylene blue (1:10) in DEPC- $\mathrm{H}_{2} \mathrm{O}$ under RNAse-free conditions. Staining was followed by LMD with a P.A.L.M. Microlaser System. Tissue fragments were collected in the adhesive lid of a microfuge tube. Immediately following LMD, tissue was removed from the lid and immersed in 100- $\mu 1$ lysis buffer (Macherey Nagel, Düren, Germany; Heinmoller et al. 2003, Niyaz et al. 2005).

\section{RNA extraction}

Total RNA from frozen tumor samples and from the LMD normal controls was isolated by homogenization of tissue in lysis buffer and purification by DNAse treatment. RNA was recovered using the RNA Kit NucleoSpin (Machery Nagel) according to the manufacturer. RNA integrity was ensured by RT-PCR of chromogranin $\mathrm{A}(\mathrm{CgA})$ and $\beta$-actin with the Qiagen one-step RT-PCR Kit by the standard protocol. CgA was used to assure the medullar origin of the normal control, as it is expressed in adrenal medulla and PC, but not expressed in adrenal cortex. Primer sequences for $C g A$ (GenBank accession number: BT006869) and $\beta$-actin (Genebank accession M10277) were as follows:

\section{CgA: forward $5^{\prime}$-GAGTGGGAGGACTC- $C A A A C G-3^{\prime}$ reverse $5^{\prime}$-CCACTTTCTCCAGCTCTG- $C C-3^{\prime}$ (Amplicon: $340 \mathrm{bp}$ ) \\ $\beta$-actin: forward $5^{\prime}$-GATGATGATATCGCC- GCGCTCGTCGTC-3' reverse 5'-GTGCCTCAGGGCAGCG- GAACCGCTCA-3' (Amplicon: 778 bp)}

After visualization of the product from the one step RT-PCR, only samples which showed expression of both were included in the further analysis.

\section{Amplification of RNA}

Two micrograms of each tumor RNA and $2 \mu \mathrm{g}$ of the pooled normal adrenal medulla RNA $(10 \times 200 \mathrm{ng})$ were amplified with the Message Amp aRNA Kit. Amino allyl cDNA was synthesized with $2 \mu \mathrm{g}$ aRNA, and then labeled and purified with the CyScribe PostLabeling Kit (Amersham Biosciences). The amino allyl-modified cDNA was chemically labeled with CyDye NHS esters. The coupling reactions of amino allyl-modified cDNA were performed separately with Cy3 and Cy5. The labeled probes were purified with Qiagen spin columns and combined before hybridization. Tumors were labeled with $\mathrm{Cy} 3$ and normal adrenal medulla with Cy5.

\section{Microarray analysis}

The combined labeled samples were hybridized to the cDNA microarray for $16 \mathrm{~h}$ at $55^{\circ} \mathrm{C}$ and washed at a stringency of $0.1 \times \mathrm{SSC} / 0.1 \% \mathrm{SDS}$ and $0.1 \times \mathrm{SSC}$. The microarray contains 11551 DNA spots from the human cDNA library 'Human Sequence-Verified cDNA UniGene Gene Sets gf200, gf201u, and gf202' (Invitrogen). Each experiment was performed as a sandwich hybridization using two arrays. cDNA microarrays were analyzed using Scan Array Express and Software from Perkin Elmer (Waltham, MA, USA).

To account for spot differences, the background corrected ratio of the two channels was calculated and then $\log 2$-transformed. Raw data were standardized to balance intensities to both dyes and to achieve comparable expression levels across experiments. A spatial and intensity-dependent standardization (Yang et al. 2002) was used to correct for inherent bias on each chip (the lowest scatter plot). The chips also contain standardized controls, which were employed to normalize and thus control for bias selected differences.

Mean log ratios were calculated from replicates, as each spot was measured twice due to the sandwich hybridization. Spots were excluded if replicates differed more than the maximum of threefold and $75 \%$ of the calculated average log ratio, or if the background intensity was higher than the signal intensity.

Differentially expressed genes were selected based on an absolute value of the $t$-statistic of 1.96 and a foldchange difference of at least 2. Prior to the cluster analysis, the expression profile of each gene was centered by subtracting the mean observed value. We performed an average linkage hierarchic clustering for genes as well as for chips with the Euclidean distance metric as implemented in the program Genesis (Sturn et al. 2002). Expression data and gene annotations 
were stored in Array Express (http://www.ebi.ac.uk/ arrayexpress/) (accession number E-MEXP-2137), which complies with minimal information about a microarray experiment (MIAME) guidelines.

\section{RT-PCR}

The mRNA was reverse transcribed into cDNA with oligo-dT primers using the Superscript 1st Strand System for RT-PCR (Invitrogen) at $42{ }^{\circ} \mathrm{C}$ for $50 \mathrm{~min}$. All PCRs were carried out on a 7500 RT-PCR System (Applied Biosystems, Foster City, CA, USA) over 40 cycles, with denaturation for $15 \mathrm{~s}$ at $95^{\circ} \mathrm{C}$ and combined annealing/extension at $60^{\circ} \mathrm{C}$ for $1 \mathrm{~min}$. Following an activation step at $95^{\circ} \mathrm{C}$ for $10 \mathrm{~min}$, determination of early growth response factor 1 and 2 (EGR 1 and 2), calsequestrin, monoaminooxidase $\mathrm{B}$ $(M A O B), S P O C K$ 2, neurogranin, and neuronatin (NNAT) mRNA expression was performed over 40 cycles with $15 \mathrm{~s}$ of denaturation at $95{ }^{\circ} \mathrm{C}$ and annealing/extension/data acquisition at $60^{\circ} \mathrm{C}$ for $60 \mathrm{~s}$ using the Power SYBR Green PCR kit (Applied Biosystems). Primer sequences were as follows:

$\begin{array}{cl}\text { EGR1: } & \text { forward } 5^{\prime} \text {-TGGGCAAGAACAC- } \\ & \text { CATGATG-3' } \\ & \text { reverse 5'-AGTTTCTCCAGAG- } \\ & \text { CTGGGTTGT-3' } \\ \text { EGR2: } & \text { forward 5'-CCCTAAAATGGTG- } \\ & \text { AATCAGAGCAT-3' } \\ & \text { reverse 5'-CGTTCCTCCCATCA- } \\ & \text { CATTGC-3' } \\ \text { Calsequestrin: } & \text { forward 5'-CTGCTAGTGAGCC- } \\ & \text { TTCCCATTT-3' } \\ & \text { reverse 5'-TATTGTTGCCCTGGC- } \\ & \text { CAATT-3' } \\ \text { MAOB: } & \text { forward 5'-GAGCCACAATAAGC- } \\ & \text { CACTGGTAT-3' } \\ & \text { reverse 5'-AGGGAGTGAAGGAG- } \\ & \text { GATAATTGG-3' } \\ \text { SPOCK2: } & \text { forward 5'-TGGATTTCCGTCTG- } \\ & \text { TAGATTTAACTG-3' } \\ & \text { reverse 5'-GGATGACTCACGG- } \\ & \text { GACTCTTG-3' } \\ & \text { forward 5'-AGCGGATCTCGG- } \\ \text { NNAT: } & \text { CAAACC-3' } \\ & \text { reverse 5'-AGCCGATGATGAG- } \\ & \text { CAGTTCAG-3' } \\ & \end{array}$

Relative fold mRNA expression levels were determined using the $2 * *\left(-\Delta \Delta C_{\mathrm{t}}\right)$ method (Livak \& Schmittgen 2001) with ribosomal protein, large, P0 $(R P L P O)$ as housekeeping control. RPLPO primer sequences were as follows: forward $5^{\prime}$-TGGGCAAGAACACCATGATG- $3^{\prime}$ and reverse $5^{\prime}$-AGTTTCTCCAGAGCTGGGTTGT-3'.

\section{Immunohistochemistry}

For immunostaining, formalin-fixed and paraffin embedded archival tumor samples and corresponding normal tissues were stained as previously described (Waldmann et al. 2008). Briefly, slides from archived ACCs were heated to $60^{\circ} \mathrm{C}$ for $1 \mathrm{~h}$, deparaffinized using xylene, and hydrated by a graded series of ethanol washes. Antigen retrieval was accomplished by microwave heating in $10 \mathrm{mM}$ sodium citrate buffer, $\mathrm{pH}$ 6.0 for $10 \mathrm{~min}$. For IHC, endogenous peroxidase activity was quenched by a 10-min incubation in $3 \%$ $\mathrm{H}_{2} \mathrm{O}_{2}$. Nonspecific binding was blocked with $10 \%$ serum for $1 \mathrm{~h}$. Sections were then probed with a primary antibody against calsequestrin (Santa Cruz Biotechnology, Santa Cruz, CA, USA) at a working dilution of 1:100 overnight at $4{ }^{\circ} \mathrm{C}$. For IHC, bound antibodies were detected using the avidin-biotin complex $(\mathrm{ABC})$ peroxidase method (ABC Elite Kit, Vector Labs, Burlingame, CA, USA). Final staining was developed with the Sigma FAST DAB peroxidase substrate kit (Sigma). As a positive control, we used murine heart tissue with every batch, as suggested by the manufacturer.

\section{Statistical analysis}

For analyzing proportions, the Mann-Whitney $U$ test was applied. In addition, a discriminant analysis (leave-one-out) was performed to estimate the diagnostic value of RT data. $P$ values $<0.05$ were considered to be statistically significant. Data were analyzed using SPSS (Chicago, IL, USA), version 14.0 for Microsoft Windows.

\section{Results}

\section{Patients}

The clinical data of five patients with benign PC and five patients with malignant PC (with DM) are shown in Table 1 . For all benign PCs, a hereditary origin was excluded by prior DHPLC analyses of the RET, VHL, $S D H B$, and $S D H D$ genes.

We conducted a gene expression profile comparing five benign and five malignant PCs to normal adrenal medulla. After background subtraction and data normalization, the ratios were averaged for all tumors. 
Table 1 Clinical data of PC which were used for microarray, RT-PCR, and IHC

\begin{tabular}{|c|c|c|c|c|c|c|}
\hline Patient ID & Histology & Age (years) & Size $(\mathrm{mm})$ & Syndrome & Ki 67 (\%) & DM \\
\hline P1 & Malignant PC & 44 & 50 & None & 1 & y LN \\
\hline P2 & Malignant PC & 42 & 95 & None & 1 & y LN \\
\hline P3 & Malignant PC & 65 & 130 & None & 5 & y LN \\
\hline P4 & Malignant PC & 78 & 50 & None & 3 & y LN \\
\hline P5 & Malignant PC & 30 & 20 & MEN2B & 1 & y LN \\
\hline P6 & Benign PC & 54 & 35 & None & ND & $\mathrm{n}$ \\
\hline P7 & Benign PC & 20 & 33 & None & 5 & $n$ \\
\hline P8 & Benign PC & 47 & 95 & None & 1 & $\mathrm{n}$ \\
\hline P9 & Benign PC & 53 & 50 & None & 1 & $\mathrm{n}$ \\
\hline P10 & Benign PC & 35 & 45 & None & 1 & $n$ \\
\hline P11 & Benign PC & 21 & 35 & MEN2A & $<1$ & $n$ \\
\hline P12 & Benign PC & 71 & 60 & None & $<1$ & $\mathrm{n}$ \\
\hline P13 & Benign PC & 48 & 75 & None & $<1$ & $n$ \\
\hline P14 & Benign PC & 48 & 40 & None & 1 & $n$ \\
\hline P15 & Benign PC & 60 & 60 & None & $<1$ & $\mathrm{n}$ \\
\hline P16 & Benign PC & 41 & 40 & None & 1 & $\mathrm{n}$ \\
\hline P17 & Benign PC & 41 & 100 & None & 2 & $\mathrm{n}$ \\
\hline P18 & Benign PC & 46 & 40 & MEN2A & $<1$ & $\mathrm{n}$ \\
\hline P19 & Malignant PG & 33 & 30 & None & 10 & y LG/LN \\
\hline P20 & Benign PC & 43 & 80 & None & ND & $\mathrm{n}$ \\
\hline P21 & Benign PC & 38 & 40 & MEN2A & $<1$ & $\mathrm{n}$ \\
\hline P22 & Benign PC & 42 & 45 & MEN2A & $<1$ & $\mathrm{n}$ \\
\hline P23 & Benign PC & 43 & 60 & None & ND & $\mathrm{n}$ \\
\hline P24 & Benign PC & 55 & 70 & None & $<1$ & $n$ \\
\hline P25 & Benign PG & 47 & 40 & None & 1 & $\mathrm{n}$ \\
\hline P26 & Benign PC & 34 & 70 & None & $<1$ & $\mathrm{n}$ \\
\hline P27 & Benign PC & 78 & 40 & None & $<1$ & $\mathrm{n}$ \\
\hline P28 & Benign PC & 62 & 75 & None & $<1$ & $\mathrm{n}$ \\
\hline P29 & Benign PC & 48 & 30 & None & $<1$ & $n$ \\
\hline P30 & Benign PC & 46 & 35 & None & $<1$ & $\mathrm{n}$ \\
\hline P31 & Malignant PG & 39 & 130 & PGS (SDHB) & 5 & y LM \\
\hline P32 & Benign PC & 47 & 95 & None & $<1$ & $\mathrm{n}$ \\
\hline P33 & Malignant PC & 20 & 45 & PGS (SDHB) & 3 & y BN, $\mathrm{P}$ \\
\hline P34 & Malignant PC & 57 & 75 & None & $<1$ & y BN \\
\hline P35 & Malignant PC & 82 & 30 & None & 1 & y LM \\
\hline P36 & Malignant PG & 37 & 20 & PGS (SDHD) & 15 & y HM \\
\hline
\end{tabular}

DM, distant metastases; PC, pheochromocytoma; PG, paraganglioma; n, no; y, yes; PGS, paraganglioma syndrome; LM, liver metastases; LN, lymph node metastases; LG, lung metastases; BN, bone metastases; P, peritoneum; HM, heart metastases; RT, real-time PCR; IHC, immunohistochemistry. For microarray $n=10$ : P1-P10; for RT $n=25: \mathrm{P} 1-\mathrm{P} 25$; and for IHC $n=20$ : P1, P3-5, P19, P31, P33-36 versus P9, P12, P15, P24, P26-30, P32.

\section{Differentially expressed genes in benign and malignant PC compared with normal adrenal medulla}

In the analysis, we found 455 spots in benign PC and 539 spots in malignant $\mathrm{PC}$ that were up-regulated more than twofold, which represent 344 and 389 unique genes respectively. A similar number of cDNA probes were down-regulated in benign (491) and malignant (674) PCs, which correspond to 411 and 572 unique genes respectively.

Among these, we identified several genes which are known to play a role in the tumorigenesis of adrenal tumors (e.g. IGF2, RGS, CDK 5, and ubiquitin).
One of the main goals of microarray analysis is to identify potential tumor suppressor genes (TSGs), which led us to focus on clearly down-regulated genes. Interestingly, MGST1 and GSTA 1,3,4 were down-regulated 62/52- and 131/84-fold in both malignant/benign $\mathrm{PC}$ respectively. Other potential TSGs were NPYIR, MAX, IGFB6, and Ezrin.

Genes up-regulated more than twofold and which potentially promote tumorigenesis were identified in malignant and benign PCs, and are enlisted in Table 2. One of the most interesting candidates was small ubiquitin-like modifier (SUMO1), which is known to be involved in the by SUMOylation of several TSGs and transcription factors, such as p53, c-jun, and NF- $\kappa \mathrm{B}$. 
Table 2 Gene sets which show different expressions in both benign and malignant pheochromocytoma (PC) compared with normal adrenal medulla

\begin{tabular}{|c|c|c|c|c|c|}
\hline Gene symbols & Gene title/definition & Gene ID & $\begin{array}{l}\text { Chromosomal } \\
\text { location }\end{array}$ & Fold change & Direction \\
\hline MGST1 & $\begin{array}{l}\text { Microsomal glutathione } \\
S \text {-transferase } 1\end{array}$ & 4257 & 12p12.3-p12.1 & $61.98 / 131.73$ & Down \\
\hline GSTA3 & Glutathione $S$-transferase $\alpha 3$ & 2940 & $6 p 12.1$ & $52.33 / 83.61$ & Down \\
\hline GPI & Glucose phosphate isomerase & 2821 & $19 q 13.1$ & $24.69 / 55.37$ & Down \\
\hline MAX & MYC associated factor $X$ & 4149 & $14 q 23$ & $18.72 / 27.15$ & Down \\
\hline$F D X 1$ & Ferredoxin 1 & 2230 & $11 q 22$ & $17.39 / 27.15$ & Down \\
\hline NPY1R & Neuropeptide Y receptor Y1 & 4886 & $4 q 31.3-q 32$ & $14.89 / 25.70$ & Down \\
\hline NUPR1 & $\begin{array}{l}\text { Nuclear protein tranascriptional } \\
\text { regul. } 1\end{array}$ & 26471 & $16 p 11.2$ & $12.07 / 15.92$ & Down \\
\hline$M A O A$ & Monoamine oxidase $\mathrm{A}$ & 4128 & Xp11.3 & $5.05 / 13.48$ & Down \\
\hline LAMA2 & Laminin, $\alpha 2$ & 3908 & $6 q 22-q 23$ & $7.69 / 9.00$ & Down \\
\hline MT1M & Metallothionein $1 \mathrm{M}$ & 4499 & $16 q 13$ & $6.87 / 10.20$ & Down \\
\hline GSTA4 & Glutathione $S$-transferase $\alpha 4$ & 2941 & $6 \mathrm{p} 12.1$ & $6.31 / 8.74$ & Down \\
\hline IGFBP6 & IGF-binding protein 6 & 3489 & $12 q 13$ & $7.05 / 8.30$ & Down \\
\hline VIL2 & Villin 2 (ezrin) & 7430 & $6 q 25.2-q 26$ & $6.32 / 7.40$ & Down \\
\hline GSTT1 & Glutathione $S$-transferase $\theta 1$ & 2952 & $22 q 11.23$ & $8.22 / 6.99$ & Down \\
\hline DNASE1L3 & Deoxyribonuclease I-like 3 & 1776 & $3 p 21.1-p 14.3$ & $7.45 / 6.34$ & Down \\
\hline$N G F R$ & Nerve growth factor receptor & 4804 & $17 q 21-q 22$ & $3.55 / 5.08$ & Down \\
\hline EGFR & Epidermal growth factor receptor & 1956 & $7 \mathrm{p} 12$ & $3.17 / 4.51$ & Down \\
\hline SP100 & SP100 nuclear antigen & 6672 & $2 q 37.1$ & $2.40 / 4.15$ & Down \\
\hline CTNNB1 & $\begin{array}{l}\text { Catenin (cadherin-associated } \\
\text { protein) }\end{array}$ & 1499 & $3 p 21$ & $3.25 / 3.30$ & Down \\
\hline SUMO1 & $\begin{array}{l}\text { SMT3 suppressor of mif two } 3 \\
\text { homolog } 1\end{array}$ & 7341 & $2 q 33$ & $14.31 / 9.99$ & Up \\
\hline IGF2 & IGF2 (somatomedin A) & 3481 & $11 \mathrm{p} 15.5$ & $12.90 / 9.65$ & Up \\
\hline RGS5 & $\begin{array}{l}\text { Regulator of G-protein } \\
\text { signaling } 5\end{array}$ & 8490 & 1q23.1 & $6.45 / 4.52$ & Up \\
\hline CDK5R2 & $\begin{array}{l}\text { Cyclin-dependent kinase } 5, \\
\text { regulatory subunit } 2 \text { (p39) }\end{array}$ & 8941 & $9 q 33$ & $4.63 / 3.81$ & Up \\
\hline UBE2D3 & $\begin{array}{l}\text { Ubiquitin-conjugating enzyme } \\
\text { E2D } 3 \text { (UBC4/5 homolog, yeast) }\end{array}$ & 7323 & $4 q 24$ & $3.86 / 3.58$ & Up \\
\hline MUM1 & $\begin{array}{l}\text { Melanoma associated antigen } \\
\text { (mutated) } 1\end{array}$ & 84939 & $19 p 13.3$ & $3.74 / 4.12$ & Up \\
\hline IGFBPL1 & IGF-binding protein-like 1 & 347252 & $9 p 13.1$ & $3.70 / 3.58$ & Up \\
\hline CSNK1G3 & Casein kinase $1, \gamma 3$ & 1456 & $5 q 23$ & $2.72 / 3.20$ & Up \\
\hline MARK3 & $\begin{array}{l}\text { MAP/microtubule affinity- } \\
\text { regulating kinase } 3\end{array}$ & 4140 & $14 q 32.3$ & $2.90 / 3.13$ & Up \\
\hline TRAF5 & $\begin{array}{l}\text { TNF receptor-associated } \\
\text { factor } 5\end{array}$ & 7188 & $1 q 32$ & $3.65 / 3.01$ & Up \\
\hline$C D C 42 B P A$ & $\begin{array}{l}\text { CDC42-binding protein } \\
\text { kinase } \alpha \text { (DMPK-like) }\end{array}$ & 8476 & $1 q 42.11$ & $3.24 / 4.69$ & Up \\
\hline
\end{tabular}

The direction of expression difference 'Down' and 'Up' indicates underexpression (Down) and overexpression (Up). The first number in the 'Fold-change' column represents malignant PC, and the second number represents benign PC. Abbreviations and full names of genes are listed as well as the chromosomal location and gene ID (NCBI online database).

\section{Differentially expressed genes in malignant compared with benign PC}

Nineteen spots were detected to be up-regulated, and 113 spots to be down-regulated with at least a twofold difference at the mRNA level in malignant PC compared with benign PC. This represents 15 and 102 unique genes. A selection of these genes is shown in Table 3.

\section{Unsupervised hierarchical cluster}

Transcriptional profiles distinguished between malignant and benign PCs and identified several differentially expressed transcripts as demonstrated by cluster analysis (as shown in Fig. 1). The majority of the differentially expressed genes were down-regulated. 
Table 3 Gene sets which distinguish between malignant and benign pheochromocytoma (PC) identified by microarray analysis

\begin{tabular}{|c|c|c|c|c|c|c|}
\hline $\begin{array}{l}\text { Gene } \\
\text { symbols }\end{array}$ & Gene title/definition & Gene ID & $\begin{array}{l}\text { Chromosomal } \\
\text { location }\end{array}$ & $\begin{array}{l}\text { Fold } \\
\text { change }\end{array}$ & Direction & $\begin{array}{l}\text { Comparison } \\
\text { (Brouwers } \\
\text { et al.) }\end{array}$ \\
\hline FOSB & $\begin{array}{l}\text { FBJ murine osteosarcoma viral oncogene } \\
\text { homolog } B\end{array}$ & 2354 & $19 q 13.32$ & 14.36 & Down & NS \\
\hline NRGN & $\begin{array}{l}\text { Neurogranin (protein kinase C substrate, } \\
\text { RC3) }\end{array}$ & 4900 & $11 \mathrm{q} 24$ & 9.63 & Down & NS \\
\hline$A R C$ & $\begin{array}{l}\text { Activity-regulated cytoskeleton-associated } \\
\text { protein }\end{array}$ & 23237 & $8 q 24.3$ & 7.21 & Down & ND \\
\hline QPCT & $\begin{array}{l}\text { Glutaminyl-peptide cyclotransferase } \\
\text { (glutaminyl cyclase) }\end{array}$ & 25797 & 2 p22.2 & 6.01 & Down & $S$ \\
\hline CCL4L2 & Chemokine (C-C motif) ligand 4-like 2 & 388372 & $17 q 12$ & 6.35 & Down & $\mathrm{S}$ \\
\hline EGR2 & $\begin{array}{l}\text { Early growth response } 2 \text { (Krox-20 homolog, } \\
\text { Drosophila) }\end{array}$ & 1959 & $10 \mathrm{q} 21.1$ & 5.56 & Down & $S$ \\
\hline RGS1 & Regulator of G-protein signaling 1 & 5996 & $1 q 31$ & 5.48 & Down & $\mathrm{S}$ \\
\hline PLAT & Plasminogen activator, tissue & 5327 & $8 p 12$ & 4.84 & Down & NS \\
\hline$M A O B$ & Monoamine oxidase B & 4129 & Xp11.23 & 4.95 & Down & NS \\
\hline PLAUR & Plasminogen activator, urokinase receptor & 5329 & $19 q 13$ & 4.60 & Down & NS \\
\hline SPOCK2 & $\begin{array}{l}\text { Sparc/osteonectin, cwcv and kazal-like } \\
\text { domains proteoglycan (testican) } 2\end{array}$ & 9806 & 10pter-q25.3 & 4.42 & Down & $\mathrm{S}$ \\
\hline GLDN & Gliomedin & 342035 & $15 q 21.2$ & 4.17 & Down & ND \\
\hline CCL2 & Chemokine (C-C motif) ligand 2 & 6347 & $17 q 11.2-q 12$ & 4.38 & Down & $\mathrm{S}$ \\
\hline EGR1 & Early growth response 1 & 1958 & $5 q 31.1$ & 4.05 & Down & $S$ \\
\hline NNAT & Neuronatin & 4826 & $20 q 11.2-q 12$ & 3.56 & Down & NS \\
\hline$C D 163 L 1$ & CD163 molecule-like 1 & 283316 & $12 p 13.3$ & 3.49 & Down & $S$ \\
\hline $\mathrm{PCDH} 20$ & Protocadherin 20 & 64881 & $13 q 21$ & 3.31 & Down & S \\
\hline H19 & $\begin{array}{l}\text { H19, imprinted maternally expressed } \\
\text { untranslated mRNA }\end{array}$ & 283120 & $11 \mathrm{p} 15.5$ & 3.15 & Down & $S$ \\
\hline FABP5 & $\begin{array}{l}\text { Fatty acid-binding protein } 5 \text { (psoriasis- } \\
\text { associated), E-FABP }\end{array}$ & 344332 & $8 q 21.13$ & 3.15 & Down & NS \\
\hline CITED2 & $\begin{array}{l}\text { Cbp/p300-interacting transactivator, with } \\
\text { Glu/Asp-rich carboxy-terminal domain, } 2\end{array}$ & 10370 & $6 q 23.3$ & 3.04 & Down & NS \\
\hline JUNB & Jun B proto-oncogene & 3726 & $19 p 13.2$ & 3.03 & Down & $\mathrm{S}$ \\
\hline DUSP5 & Dual specificity phosphatase 5 & 1847 & $10 q 25$ & 2.85 & Down & $S$ \\
\hline SERPING1 & $\begin{array}{l}\text { Serpin peptidase inhibitor, clade } G \\
\text { (C1 inhibitor), member } 1\end{array}$ & 710 & $11 q 12-q 13.1$ & 2.82 & Down & NS \\
\hline$M A O A$ & Monoamine oxidase $A$ & 4128 & p11.3 & 2.67 & Down & NS \\
\hline BIN1 & Bridging integrator 1 & 274 & $2 q 1$ & 2.40 & Down & $S$ \\
\hline AIFM1 & $\begin{array}{l}\text { Apoptosis-inducing factor, mitochondrion- } \\
\text { associated, } 1\end{array}$ & 9131 & Xq25-q26 & 2.38 & Down & NS \\
\hline $\mathrm{CDH} 2$ & Cadherin 2 , type $1, \mathrm{~N}$-cadherin (neuronal) & 1000 & $18 q 11.2$ & 2.22 & Down & $S$ \\
\hline$D K K 3$ & Dickkopf homolog 3 (Xenopus laevis) & 27122 & $11 \mathrm{p} 15.2$ & 2.12 & Down & NS \\
\hline$C D C 26$ & $\begin{array}{l}\text { Cell division cycle } 26 \text { homolog } \\
\text { (Saccharomyces cerevisiae) }\end{array}$ & 246184 & $9 q 32$ & 2.11 & Down & $\mathrm{S}$ \\
\hline FOS & $\begin{array}{l}\text { V-fos FBJ murine osteosarcoma viral } \\
\text { oncogene homolog }\end{array}$ & 2353 & $14 q 24.3$ & 2.11 & Down & S \\
\hline IGFBP2 & IGF-binding protein & 3485 & $2 q 33-q 34$ & 2.11 & Down & ND \\
\hline CTSO & Cathepsin O & 1519 & $4 q 31-q 32$ & 2.09 & Down & ST \\
\hline SEZ6 & Seizure-related 6 homolog (mouse) & 124925 & $17 q 11.2$ & 5.57 & Up & NS \\
\hline CASQ2 & Calsequestrin 2 (cardiac muscle) & 845 & 1p13.3-p11 & 3.63 & Up & NS \\
\hline STXBP6 & Syntaxin-binding protein 6 (amisyn) & 29091 & $14 q 12$ & 2.91 & Up & NS \\
\hline RNF32 & Ring finger protein 32 & 140545 & $7 q 36$ & 2.47 & Up & $S$ \\
\hline MAN1A2 & Mannosidase, $\alpha$, class $1 \mathrm{~A}$, member 2 & 10905 & $1 p 13$ & 2.27 & Up & NS \\
\hline DTWD1 & DTW domain containing 1 & 56986 & $15 . q 21.2$ & 2.22 & Up & ST \\
\hline MINA & MYC-induced nuclear antigen & 84864 & $3 q 11.2$ & 2.10 & Up & $S$ \\
\hline
\end{tabular}

The direction of expression difference 'Down' and 'Up' indicates underexpression (Down) and overexpression (Up). Abbreviations and full names of genes are listed as well as chromosomal location and gene ID (NCBI online database). S, significant; NS, not significant; ST, statistical trend $(P<0.1)$. 


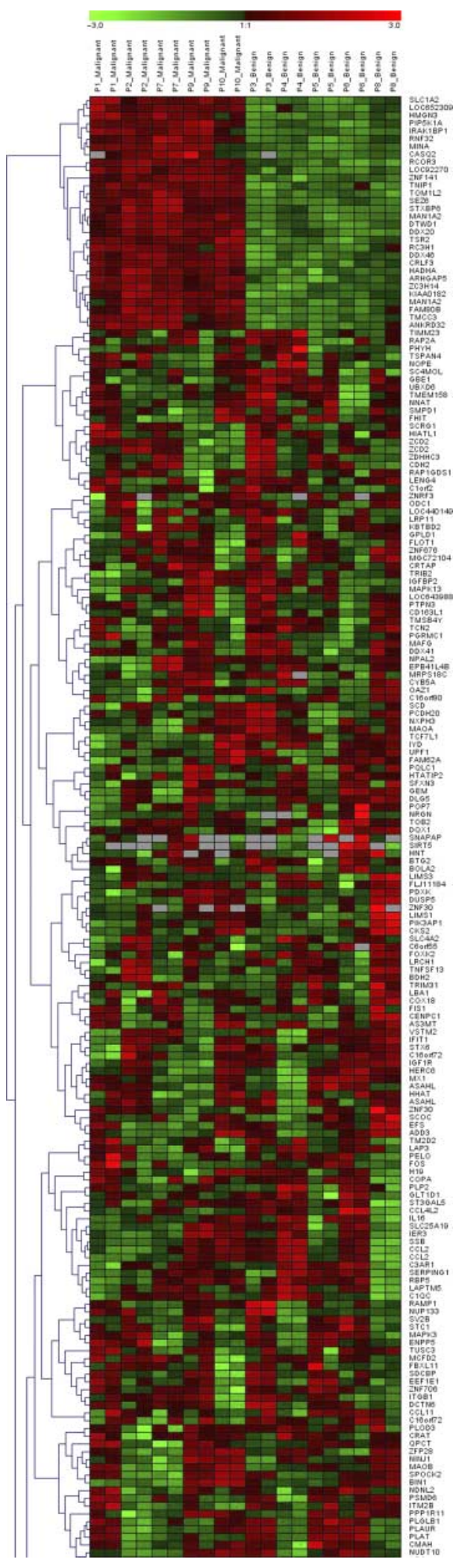

\section{Analysis of datatset with gene set enrichment analysis (GSEA), DAVID, and EASE}

We identified 50 gene sets, which discriminate between malignant and benign PC with a $P$ value $<0.01$. If we reset the significance level to a $P$ value of 0.05 , the number of gene sets increased to 135 . A core enrichment was shown for eight gene sets: ZNF198, UBE2N, CHR17P11, BRACX UP, CSNK1A1, WNT pathway, and $S U F U$. Related to the Wnt pathway, TSGs (CREBBP), kinases (GSK3 $\beta, N L K$, and MAP3K7), oncogenes (CTNNB1, MYC, and CCND1), and transcription factors (TCF1, HDACl, and PPARD) were shown to be significantly overexpressed in malignant PC.

\section{Validation of microarray analysis by RT-PCR}

Some of the genes which were differentially expressed in malignant and benign PC were subjected to further analysis to ensure the validity of the microarray. Expression levels of calsequestrin, neurogranin, NNAT, SPOCK2, EGR2, and MAOB were confirmed by RT-PCR in 6 malignant and 19 benign PCs (P1-P25) as shown in Fig. 2. ERCC1, EGR1, and MAOA did not reveal statistically different levels of expression in malignant and benign PC. $P$ values were calculated by the Mann-Whitney test, and are shown in Fig. 2. The discriminant analysis (leave-one-out) showed that $78 \%$ of cross-validated grouped cases were classified correctly.

\section{Comparison with the study of Brouwers et al.}

A comparison of the gene lists from both microarrays revealed a total of $4709 / 15233(30.9 \%)$ genes that matched. Brouwers et al. found a total of 1126 genes that were up-regulated, and 1633 that were downregulated in all assessed malignant $\mathrm{PC}$ compared with benign PC. In primary metastatic/malignant PC, Brouwers et al. found a total of 1263 genes that were up-regulated, and 1808 genes that were downregulated versus benign PC. The overall conformance of Brouwers et al. and the present microarray analysis is illustrated by a Venn diagram in Fig. 3. The group of

Figure 1 Supervised hierarchical cluster in malignant and benign pheochromocytomas. On the left, malignant PCs (P1, $\mathrm{P} 2, \mathrm{P} 7, \mathrm{P}$, and $\mathrm{P} 10$ ) and on the right, benign PCs (P3, P4, P5, $P 6$, and $P 8$ ) are shown. Expression differences are indicated by the color (red, overexpressed (threefold difference); green, underexpressed (threefold difference) compared with the average expression of all samples). Genes that revealed a differential expression in malignant and benign $\mathrm{PC}$ by the microarray analysis are listed in the right margin. Full colour version of this figure available via http://dx.doi.org/10.1677/ ERC-09-0118. 

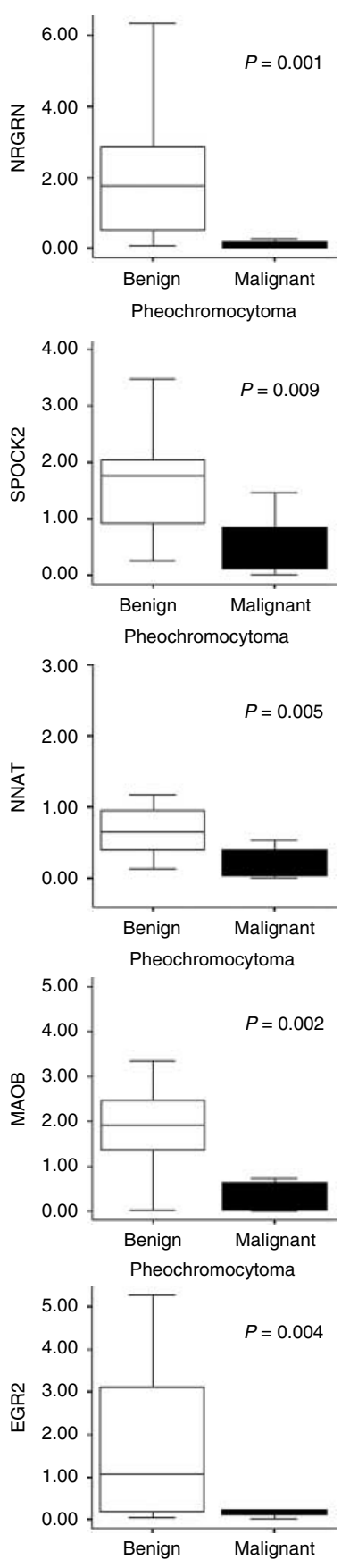

Pheochromocytoma

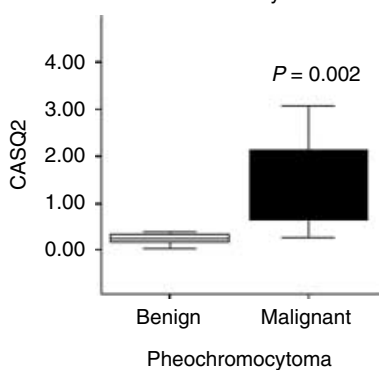

Pheochromocytoma all malignant PCs is referred as Brouwers_1 in Fig. 3 (yellow), while the primary malignant PCs are referred as Brouwers_4 in Fig. 3 (purple). This takes into account that a subgroup of PC patients present with DM at diagnosis (Brouwers_4), while others develop metastases in further follow-up (these are included in Brouwers_1). The meaning of the color code is explained in detail in the legend of Fig. 3. Details on the comparison with Brouwers data for distinct genes are given in Table 3, which either shows confirmation (S, significant) or difference (NS, not significant) of the two datasets. Eighteen of the 39 genes (present on both chips) were significantly differentially expressed in both analyses (see also Table 3). Interestingly, 5/11 down-regulated genes that overlapped are cation binders (secreted protein acidic and rich in cysteine (SPARC), SP100 nuclear antigen, vav2 oncogene, $E M R 3, R C H Y 1$ ), and 5/11 genes are known to play role in the cell cycle (SPARC, SP100 nuclear antigen, vav2 oncogene, NFARC1, p78). It has to be kept in mind that only 704/1962 (35.9\%) of up-regulated genes and $886 / 2691$ (32\%) of down-regulated genes of the total NIH dataset (number in parentheses) were found on the chip used for the present study.

\section{IHC for calsequestrin}

IHC revealed an increased expression of calsequestrin at the protein level in ten malignant PCs (P1, P3-5, P19, $\mathrm{P} 31$, and $\mathrm{P} 33-36)$ compared with ten benign PCs (P9, P12, P15, P24, P26-30, and P32). Whereas only one of the ten benign PCs showed strong calsequestrin expression, seven of ten malignant PCs did $(P=0.01)$. Clinical data are summarized in Tables 1 and 3. Positive staining was localized to the cytoplasm as in the positive control (murine heart muscle). Figure 4 shows the typical expression difference in a benign and a malignant PC. Sustenacular cells stained positive in malignant and benign PC, and were used as internal control.

\section{Discussion}

Malignancy in PC can only be established accurately if DM are present. Neither histology nor clinical patterns can distinguish between patients with truly benign PC and patients who will develop DM in further follow-up.

Figure 2 Comparative quantification of calsequestrin (CASQ2), neurogranin (NGRN), Sparc, EGR2, MAOB, and Sez6 in 25 $\mathrm{PCs}$ ( 6 malignant/19 benign) by $\mathrm{RT}$. The relative expression levels of the above mentioned genes are shown as whisker plots. The left bar of each diagram stands for benign PC, and the right bar stands for malignant PC. The statistical significance ( $t$-test) is included in each diagram. 
Comparison of the NIH and own dataset

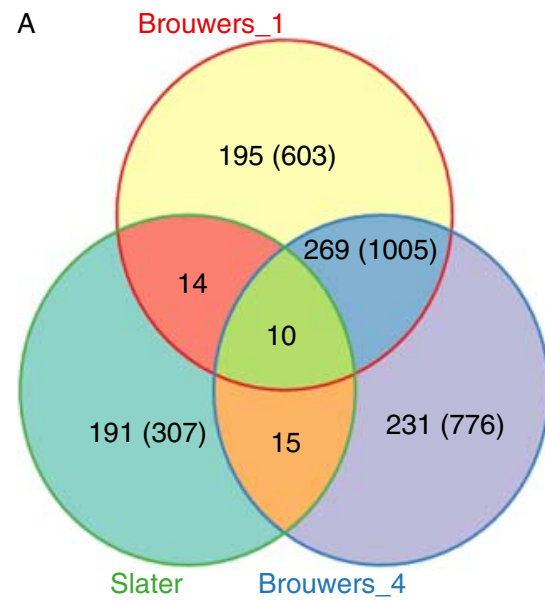

Underexpression in malignant PC

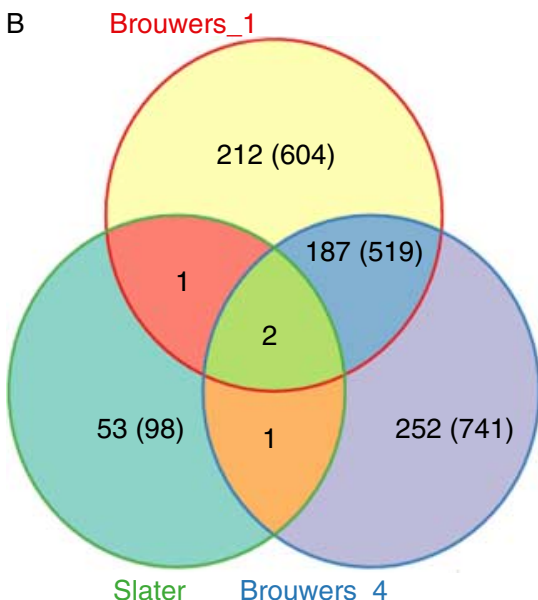

Overexpression in malignant PC

Figure 3 Venn diagram with the overlap between the microarray of the present study and the NIH dataset (Brouwers et al. 2006). On the left diagram underexpressed genes in malignant PC, and on the right overexpressed genes in malignant PC are shown. Brouwers 1 and 4 represent all malignant tumors (1) and all primary malignant tumors (4). Slater represents data of the present study. Colors (yellow, purple, and turquoise) are assigned to genes, which are underexpressed or overexpressed without an intersection between the three groups (Slater, Brouwers_1 and 4). Overlapping genes are indicated by the colors orange, green, red, and blue. The number of genes which are assessed by both chips (Slater/Brouwers) are shown without parentheses. If genes were only on the array of Brouwers et al., number is given in parentheses. Full colour version of this figure available via http://dx.doi.org/10.1677/ERC-09-0118.

DM in patients with apparently benign PC occur in up to $7 \%$ of cases (Khorram-Manesh et al. 2005). The treatment of metastatic PC is an unresolved problem as response to chemotherapy, radiotherapy, and I131-MIBG is very limited and results in 5-year survival rate of $\sim 40 \%$ (Remine et al. 1974, Safford et al. 2003, Khorram-Manesh et al. 2005). However, long-term survival of more than 20 years has been reported, reflecting the different biological subtypes of malignant PCs (Jarvelainen \& Viikari 2001, Yoshida et al. 2001).

Gene expression profiling is an attractive approach to obtain detailed insights into the biology of PC. It offers a possibility to identify both molecular markers for malignancy and potential targets for novel therapies. Therefore, several studies have assessed malignant and benign PC by gene expression profiling (Anouar et al. 2006, Brouwers et al. 2006, Thouennon et al. 2007).

In our study, we identified a set of 130 genes, which could distinguish between malignant and benign PC. To validate our results, we confirmed the differential expression by RT-PCR, and we increased the sample size to $29 \mathrm{PCs}$, including the ten PCs, which were subjected to the microarray analysis.

In addition, an unsupervised clustering clearly could discriminate malignant from benign PC while blinded to clinical characteristics. This indicates that gene expression profiling may serve as an approach to classify PC and to evaluate prognosis. Based on expression data, patients can be subjected to closer follow-up or adjuvant therapy in the future.

Our study shows that the majority of genes were under-expressed in malignant PC (119/132), which is in the line with previous studies (Anouar et al. 2006, Brouwers et al. 2006, Thouennon et al. 2007). Interestingly, several of these genes encode neuroendocrine factors (neurogranin and NNAT), enzymes involved in catecholamine metabolism (MAOA) and peptide processing (QPCT, SERPING1). The decreased expression may reflect a loss of differentiation in malignant PC, which possibly arises from immature neuroendocrine precursor cells. According to Nakamura \& Kaelin (2006), neural crest precursor cells fail to undergo apoptosis during embryonic development. This is supported by the findings of Powers et al. (2007) who reported a neural progenitor profile in NF1 knock out mice PCs.

With SPARC, protocadherin 20 (PCDH20), and $\mathrm{N}$-cadherin, we identified matricellular proteins that are known regulators of metastasis in the context of epithelial-to-mesenchymal transition (EMT). SPARC was down-regulated in malignant PC, which is consistent with its role in the inhibition of angiogenesis and tumor growth in neuroblastomas (Chlenski et al. 2002, 2006). Powers found SPARC to be up-regulated 

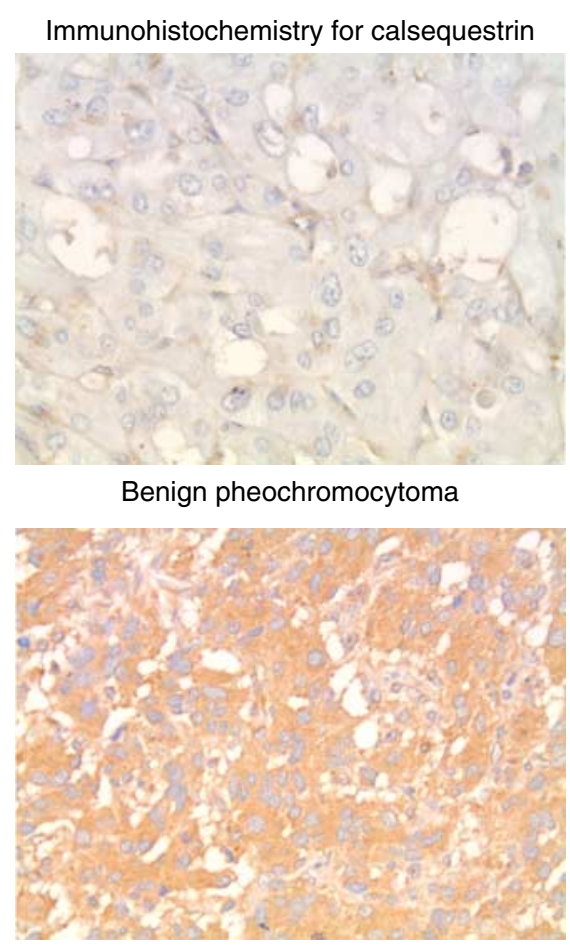

Malignant pheochromocytoma

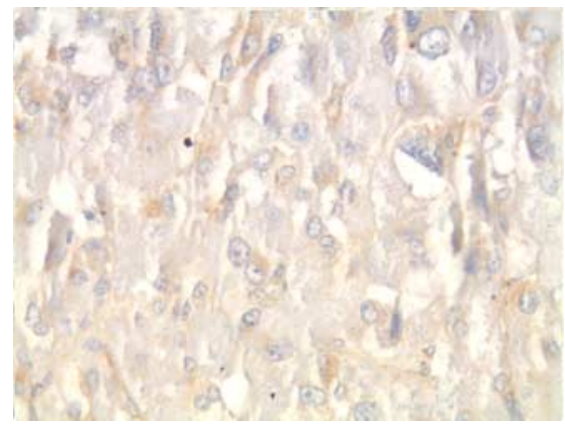

Benign pheochromocytoma

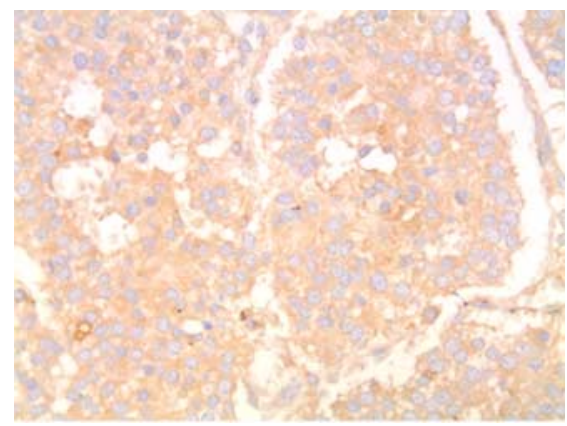

Malignant pheochromocytoma

Figure 4 Immunostaining for calsequestrin in two malignant and two benign PCs. Immunostaining for calsequestrin in two different beingn and malignant PCs. Note the intense staining (brown) in malignant PC compared with benign PC. Nonneoplastic sustentacular cells were stained in both malignant and benign PCs. Full colour version of this figure available via http://dx.doi.org/10.1677/ERC-09-0118. in PC compared with normal adrenal medulla (11.1-fold) as did we in our study (3.9-fold, data not shown; Powers et al. 2007). N-cadherin was also found to be down-regulated in a neuroblastoma cell line, and is a target gene of RASSF1A (Agathanggelou et al. 2003). $P C D H 2 O$ belongs to a subfamily of the cadherin superfamily, displays features of a TSG, and is found to be down-regulated in small cell lung cancer (Imoto et al. 2006). Also, an activation of the WNT pathway might indicate a role of EMT in the malignant transformation of PC, as activation of the $\mathrm{WNT} / \beta$-catenin pathway is known to provoke EMT in colorectal cancer (Brabletz et al. 2005).

Seizure-related 6 homolog (SEZ6) was one of the few up-regulated genes in malignant PC, and is reported to serve as a prognostic marker for lung cancer (Ishikawa et al. 2006). Calsequestrin was also up-regulated in malignant PC, as confirmed by RT and IHC (Fig. 4). It is normally expressed in heart muscle, and plays role in subcellular calcium storage. The function in the context of PC is unknown, but one could speculate that an impaired calcium homeostasis might influence intracellular signaling.

Two candidates for TSGs are BIN1 and NNAT, which are both under-expressed in malignant PC. NNAT is overexpressed in neuroblastoma with a favorable diagnosis, while BIN1 expression is reported to be reduced in neuroblastomas with unfavorable biological features (Tajiri et al. 2003, Higashi et al. 2007). Interestingly, we found SUMO, a smallubiquitin-like modifier, to be up-regulated in benign and malignant PC. SUMO controls diverse cellular functions of protein targets including transcription factors and other regulators that maintain cellular homeostasis. Additionally, SUMO plays important roles in genomic stability and DNA repair (Kim \& Baek 2006).

Chromosomal losses of $6 \mathrm{q}$ and $17 \mathrm{p}$ are frequently observed in malignant PC (Dannenberg et al. 2000). Focusing on chromosomal locations of genes that showed decreased expression in malignant PC, we identified CITED 2 and phosphatase and actin regulator 2 (PHACTR2) on chromosome 6q, but none on chromosome $17 \mathrm{p}$.

A limitation of this study is the fact that some of the apparently benign PCs that were used for the microarray may turn out to be malignant in further follow-up. As reported in the literature, DM can develop 20 years after surgery (Tanaka et al. 1993), and only lifelong follow-up can assure that the PC is truly benign. Another limitation is the small sample number, which underlines the ultimate need for 
multicenter studies. To reevaluate our data, we compared it with the data of Brouwers et al. (2006). Thirty-nine of 191 (20\%) down-regulated genes were also decreased expressed in Brouwers analysis (see Fig. 3), while only $4 / 55$ (7\%) of up-regulated genes were confirmed by Brouwers. The limited comparibility (different array platforms) must be carefully considered when interpretate these results. Only $\sim 30 \%$ of genes assessed by our array platform were also found on the array chip used by Brouwers et al. Different array platforms (affymetrix versus agilant) were applied, and therefore, their numeric results could not be directly combined to select significant markers. The resulting gene sets were therefore generated by simple intersection on their Unigene symbol annotation. At least, the normal adrenal medulla was obtained from patients who underwent adrenalctomy due to adrenocortical disease (primary hypercortisolism/ primary hyperaldosteronism). Thus, effects of cortisol on gene expression in the adrenal medulla have only been reported so far for tyrosinhydroxylase and catecholamin synthesizing enzymes, this may have influenced our data (Kvetnansky et al. 1995). Under different circumstances, where adrenal medulla could be obtained (Organ donor, cancer-associated nephrectomy), changes in gene expression appear to be likely, as even histological have been described (Gelfman 1964, Cuschieri 1969, Masterson \& Mostafa 1998).

Future studies should validate marker genes and elaborate pathways by functional studies. The development of tissue microarrays with paraffin-embedded PC samples would provide an instrument for testing different prognostic markers when clinical data on follow-up, especially the development of DM, is available.

In conclusion, we performed a microarray analysis to discriminate between malignant and benign $\mathrm{PC}$ and identified a set of genes that clearly classify PC. This study offers a useful database for further elaboration of prognostic markers in PCs, and provides insights into the biology of this disease.

\section{Declaration of interest}

The authors declare that there is no conflict of interest that could be perceived as prejudicing the impartiality of the research reported.

\section{Funding}

This research did not receive any specific grant from any funding agency in the public, commercial, or not-forprofit sector.

\section{Acknowledgements}

We thank Brunhilde Chaloupka for excellent technical assistance and Michael Krause for assistance with the F-chip. In addition, we thank Prof Pacak and Dr Brouwers from the NIH who made their microarray data available to us. Florian Finkernagel helped us with the comparative statistical analysis of the NIH dataset.

\section{References}

Agathanggelou A, Biéche I, Ahmed-Choudhury J, Nicke B, Dammann R, Baksh S, Gao B, Minna JD, Downward J, Maher ER et al. 2003 Identification of novel gene expression targets for the Ras association domain family 1 (RASSF1A) tumor suppressor gene in non-small cell lung cancer and neuroblastoma. Cancer Research 63 5344-5351.

Amar L, Servais A, Gimenez-Roqueplo AP, Zinzindohoue F, Chatellier G \& Plouin PF 2005 Year of diagnosis, features at presentation, and risk of recurrence in patients with pheochromocytoma or secreting paraganglioma. Journal of Clinical Endocrinology and Metabolism 90 2110-2116.

Anouar Y, Yon L, Guillemot J, Thouennon E, Barbier L, Gimenez-Roqueplo AP, Bertherat J, Lefebvre H, Klein M, Muresan M et al. 2006 Development of novel tools for the diagnosis and prognosis of pheochromocytoma using peptide marker immunoassay and gene expression profiling approaches. Annals of the New York Academy of Sciences 1073 533-540.

Beard CM, Sheps SG, Kurland LT, Carney JA \& Lie JT 1983 Occurrence of pheochromocytoma in Rochester, Minnesota, 1950 through 1979. Mayo Clinic Proceedings 58 802-804.

Brabletz T, Hlubek F, Spaderna S, Schmalhofer O, Hiendlmeyer E, Jung A \& Kirchner T 2005 Invasion and metastasis in colorectal cancer: epithelial-mesenchymal transition, mesenchymal-epithelial transition, stem cells and beta-catenin. Cells, Tissues, Organs 179 56-65.

Brouwers FM, Elkahloun AG, Munson PJ, Eisenhofer G, Barb J, Linehan WM, Lenders JW, De Krijger R, Mannelli M, Udelsman R et al. 2006 Gene expression profiling of benign and malignant pheochromocytoma. Annals of the New York Academy of Sciences 1073 541-556.

Chlenski A, Liu S, Crawford SE, Volpert OV, DeVries GH, Evangelista A, Yang Q, Salwen HR, Farrer R, Bray J et al. 2002 SPARC is a key Schwannian-derived inhibitor controlling neuroblastoma tumor angiogenesis. Cancer Research 62 7357-7363.

Chlenski A, Liu S, Guerrero LJ, Yang Q, Tian Y, Salwen HR, Zage P \& Cohn SL 2006 SPARC expression is associated with impaired tumor growth, inhibited angiogenesis and changes in the extracellular matrix. International Journal of Cancer 118 310-316.

Cuschieri A 1969 The morpholgy of adrenal glands in advanced breast cancer. British Journal of Surgery $\mathbf{5 6}$ $822-826$. 
Dahia PL, Ross KN, Wright ME, Hayashida CY, Santagata S, Barontini M, Kung AL, Sanso G, Powers JF, Tischler AS et al. 2005 A HIF1alpha regulatory loop links hypoxia and mitochondrial signals in pheochromocytomas. PLoS Genetics 1 72-80.

Dannenberg H, Speel EJ, Zhao J, Saremaslani P, van Der Harst E, Roth J, Heitz PU, Bonjer HJ, Dinjens WN, Mooi WJ et al. 2000 Losses of chromosomes 1p and 3q are early genetic events in the development of sporadic pheochromocytomas. American Journal of Pathology 157 353-359.

Eisenhofer G, Bornstein SR, Brouwers FM, Cheung NK, Dahia PL, de Krijger RR, Giordano TJ, Greene LA, Goldstein DS, Lehnert H et al. 2004a Malignant pheochromocytoma: current status and initiatives for future progress. Endocrine-Related Cancer 11 423-436.

Eisenhofer G, Huynh TT, Pacak K, Brouwers FM, Walther MM, Linehan WM, Munson PJ, Mannelli M, Goldstein DS \& Elkahloun AG 2004b Distinct gene expression profiles in norepinephrine- and epinephrine-producing hereditary and sporadic pheochromocytomas: activation of hypoxia-driven angiogenic pathways in von HippelLindau syndrome. Endocrine-Related Cancer 11 897-911.

Elkahloun AG, Powers JF, Nyska A, Eisenhofer G \& Tischler AS 2006 Gene expression profiling of rat pheochromocytoma. Annals of the New York Academy of Sciences 1073 290-299.

Gelfman NA 1964 Morphologic changes of adrenal cortex in disease. Yale Journal of Biology and Medicine 37 31-54.

Gimenez-Roqueplo AP, Favier J, Rustin P, Rieubland C, Crespin M, Nau V, Khau Van Kien P, Corvol P, Plouin PF, Jeunemaitre X et al. 2003 Mutations in the SDHB gene are associated with extra-adrenal and/or malignant phaeochromocytomas. Cancer Research 63 5615-5621.

Heinmoller E, Bockholt A, Werther M, Ziemer M, Müller A, Ghadimi BM \& Rüschoff J 2003 Laser microdissection of small tissue samples - application to chronic pancreatitis tissues. Pathology, Research and Practice 199 363-371.

Higashi M, Tajiri T, Kinoshita Y, Tatsuta K, Souzaki R, Maehara Y, Suita S \& Taguchi T 2007 High expressions of neuronatin isoforms in favorable neuroblastoma. Journal of Pediatric Hematology/Oncology 29 551-556.

Imoto I, Izumi H, Yokoi S, Hosoda H, Shibata T, Hosoda F, Ohki M, Hirohashi S \& Inazawa J 2006 Frequent silencing of the candidate tumor suppressor PCDH20 by epigenetic mechanism in non-small-cell lung cancers. Cancer Research 66 4617-4626.

Ishikawa N, Daigo Y, Takano A, Taniwaki M, Kato T, Tanaka S, Yasui W, Takeshima Y, Inai K, Nishimura H et al. 2006 Characterization of SEZ6L2 cell-surface protein as a novel prognostic marker for lung cancer. Cancer Science 97 737-745.

Jarvelainen H \& Viikari J 2001 Malign pheochromocytoma behaving as a benign tumor. Duodecim 117 2045-2050.
Khorram-Manesh A, Ahlman H, Nilsson O, Oden A \& Jansson S 2004 Mortality associated with pheochromocytoma in a large Swedish cohort. European Journal of Surgical Oncology 30 556-559.

Khorram-Manesh A, Ahlman H, Nilsson O, Friberg P, Odén A, Stenström G, Hansson G, Stenquist O, Wängberg B, Tisell LE et al. 2005 Long-term outcome of a large series of patients surgically treated for pheochromocytoma. Journal of Internal Medicine 258 55-66.

Kim KI \& Baek SH 2006 SUMOylation code in cancer development and metastasis. Molecules and Cells 22 247-253.

Kvetnansky R, Pacak K, Fukuhara K, Viskupic E, Hiremagalur B, Nankova B, Goldstein DS, Sabban EL \& Kopin IJ 1995 Sympathoadrenal system in stress. Interaction with the hypothalamic-pituitray-adrenocortical system. Annals of the New York Academy of Sciences $\mathbf{7 7 1}$ 131-158.

Livak KJ \& Schmittgen TD 2001 Analysis of relative gene expression data using real-time quantitative PCR and the 2(-Delta Delta C(T)) method. Methods 25 402-408.

Masterson GR \& Mostafa SM 1998 Adrenocortical function in critical illness. British Journal of Anaesthesia $\mathbf{8 1}$ 308-310.

McNeil A, Blok BH, Koelmeyer TD, Burke MP \& Hilton JM 2000 Pheochromocytomas discovered during coronial autopsies in Sydney, Melbourne ans Auckland. Australian and New Zealand Journal of Medicine 30 648-652.

Melicow MM 1977 One hundred cases of pheochromocytoma (107 tumors) at the Columbia-Presbyterian Medical Center, 1926-1976: a clinicopathological analysis. Cancer 40 1987-2004.

Nakamura E \& Kaelin WG Jr 2006 Recent insights into the molecular pathogenesis of pheochromocytoma and paraganglioma. Endocrine Pathology 17 97-106.

Neumann HP, Bausch B, McWhinney SR, Bender BU, Gimm O, Franke G, Schipper J, Klisch J, Altehoefer C, Zerres K et al. 2002 Germ-line mutations in nonsyndromic pheochromocytoma. New England Journal of Medicine 346 1459-1466.

Niyaz Y, Stich M, Sägmüller B, Burgemeister R, Friedemann G, Sauer U, Gangnus R \& Schütze K 2005 Noncontact laser microdissection and pressure catapulting: sample preparation for genomic, transcriptomic, and proteomic analysis. Methods in Molecular Medicine 114 1-24.

Omura M, Saito J, Yamaguchi K, Kakuta Y \& Nishikawa T 2004 Prospective study on the prevalence of secondary hypertension among hypertensive patients visiting a general outpatient clinic in Japan. Hypertension Research 27 193-202.

Pacak K, Linehan WM, Eisenhofer G, Walther MM \& Goldstein DS 2001 Recent advances in genetics, diagnosis, localization, and treatment of pheochromocytoma. Annals of Internal Medicine 134 315-329. 
Platts J, Drew PJ \& Harvey JN 1995 Death from pheochromocytoma: lesson from a post-mortem survey. Journal of the Royal College of Physicians of London 29 299-306.

Powers JF, Evinger MJ, Zhi J, Picard KL \& Tischler AS 2007 Pheochromocytomas in Nf1 knockout mice express a neural progenitor gene expression profile. Neuroscience 147 928-937.

Proye C, Vix M, Goropoulos A, Kerlo P \& Lecomte-Houcke M 1992 High incidence of malignant pheochromocytoma in a surgical unit. 26 cases out of 100 patients operated from 1971 to 1991. Journal of Endocrinological Investigation 15 651-663.

Remine WH, Chong GC, Van Heerden JA, Sheps SG \& Harrison EG Jr 1974 Current management of pheochromocytoma. Annals of Surgery 179 740-748.

Safford SD, Coleman RE, Gockermann JP, Moore J, Feldmann JM, Leight GS, Tyler GS \& Olson JE 2003 Iodine-131 metaiodobenzylguanidine is an effective treatment for malignant pheochromocytoma and paraganglioma. Surgery 134 956-962.

Sinclair A, Isles CG, Brown I, Cameron H \& Murray GD 1987 Secondary hypertension in a blood pressure clinic. Archives of Internal Medicine 147 1289-1293.

Sturn A, Quackenbush J \& Trajanoski Z 2002 Genesis: cluster analysis of microarray data. Bioinformatics 18 207-208.

Tajiri T, Liu X, Thompson PM, Tanaka S, Suita S, Zhao H, Maris JM, Prendergast GC \& Hogarty MD 2003 Expression of a MYCN-interacting isoform of the tumor suppressor BIN1 is reduced in neuroblastomas with unfavorable biological features. Clinical Cancer Research 9 3345-3355.

Tanaka S, Ito T, Tomoda J, Higashi T, Yamada G \& Tsuji T 1993 Malignant pheochromocytoma with hepatic metastasis diagnosed 20 years after resection of the primary adrenal lesion. Internal Medicine $\mathbf{3 2}$ 789-794.

Thouennon E, Elkahloun AG, Guillemot J, GimenezRoqueplo AP, Bertherat J, Pierre A, Ghzili H, Grumolato L, Muresan M, Klein M et al. 2007 Identification of potential gene markers and insights into the pathophysiology of pheochromocytoma malignancy. Journal of Clinical Endocrinology and Metabolism 92 4865-4872.

Waldmann J, Feldmann G, Slater EP, Langer P, Buchholz M, Ramaswamy A, Saeger W, Rothmund M \& Fendrich V 2008 Expression of the zinc-finger transcription factor Snail in adrenocortical carcinoma is associated with decreased survival. British Journal of Cancer 99 1900-1907.

Yang YH, Dudoit S, Luu P, Lin DM, Peng V, Ngai J \& Speed TP 2002 Normalization for cDNA microarray data: a robust composite method addressing single and multiple slide systematic variation. Nucleic Acids Research 30 e15.

Yoshida S, Hatori M, Noshiro T, Kimura N \& Kokubun S 2001 Twenty-six-years' survival with multiple bone metastasis of malignant pheochromocytoma. Archives of Orthopaedic and Trauma Surgery 121 598-600. 